\title{
Antenatal Maternal Anxiety is Related to HPA-Axis Dysregulation and Self-Reported Depressive Symptoms in Adolescence: A Prospective Study on the Fetal Origins of Depressed Mood
}

\author{
Bea RH Van den Bergh*,', Ben Van Calster ${ }^{2}$, Tim Smits ${ }^{3}$, Sabine Van Huffel ${ }^{2}$ and Lieven Lagae ${ }^{4}$ \\ 'Department of Psychology, Katholieke Universiteit Leuven (KU Leuven), Tiensestraat, Leuven, Belgium; ${ }^{2}$ Department of Electrical Engineering \\ (ESAT-SISTA), Katholieke Universiteit Leuven (KU Leuven), Kasteelpark Arenberg, Leuven, Belgium; ${ }^{3}$ Centre for Ethics, Katholieke Universiteit \\ Leuven (KU Leuven), Deberiotstraat, Leuven, Belgium; ${ }^{4}$ Department of Paediatric Neurology, Katholieke Universiteit Leuven (KU Leuven), \\ Herestraat, Leuven, Belgium
}

\begin{abstract}
Depressive symptomatology can proceed from altered hypothalamic-pituitary-adrenocortex (HPA)-axis function. Some authors stress the role that early life stress (ELS) may play in the pathophysiology of depressive symptoms. However, the involvement of the HPA-axis in linking prenatal ELS with depressive symptoms has not been tested in a prospective-longitudinal study extending until after puberty in humans. Therefore, we examined whether antenatal maternal anxiety is associated with disturbances in HPA-axis regulation and whether the HPA-axis dysregulation mediates the association between antenatal maternal anxiety and depressive symptoms in post-pubertal adolescents. As part of a prospective-longitudinal study, we investigated maternal anxiety at 12-22, 23-32, and 32-40 weeks of pregnancy (wP) with the State Trait Anxiety Inventory (STAI). In the 14-15-year-old offspring $(n=58)$ HPA-axis function was measured through establishing a saliva cortisol day-time profile. Depressive symptoms were measured with the Children's Depression symptoms Inventory (CDI). Results of regression analyses showed that antenatal exposure to maternal anxiety at 12-22 wp was in both sexes associated with a high, flattened cortisol day-time profile $(P=0.0463)$ which, in female adolescents only, was associated with depressive symptoms $(P=0.0077)$. All effects remained after controlling for maternal smoking, birth weight, obstetrical optimality, maternal postnatal anxiety and puberty phase. Our prospective study demonstrates, for the first time, the involvement of the HPA-axis in the link between antenatal maternal anxiety/prenatal ELS and depressive symptoms for post-pubertal female adolescents. Neuropsychopharmacology (2008) 33, 536-545; doi: I 0. I038/sj.npp. I 30I450; published online 16 May 2007
\end{abstract}

Keywords: fetal programming; HPA-axis; cortisol; depressive disorder; adolescence; antenatal maternal anxiety

\section{INTRODUCTION}

Depressive disorders are among the most common psychiatric disorders, with prevalence estimates for major depressive disorder (MDD) ranging from $5 \%$ to a maximum of $20 \%$. After puberty, a maturational and potentially stressful period, the risk of developing depression increases and the prevalence among females becomes one and a half to three times that of males (Angold et al, 1998; Ge et al, 2006; Hankin and Abramson, 2001; Hankin, 2006; Kessler, 2003).

Early-life exposure to adverse environmental cues during critical windows of time in the prenatal and/or early

*Correspondence: Professor BRH Van den Bergh, Department of Psychology, University of Leuven (KU Leuven), Tiensestraat 102, 3000 Leuven, Belgium, Tel: + 3216 325860, Fax: + 3216326055 ,

E-mail: bea.vandenbergh@psy.kuleuven.be

Received 30 August 2006; revised and accepted 9 April 2007 postnatal life period (eg, nutrient restriction or maternal anxiety) could predispose the individual for somatic and mental diseases. This especially holds for stress-related disorders such as depression in which HPA-axis dysregulation plays a pathophysiological role. The above is in line with the 'fetal (or developmental) programming-hypothesis' which has been tested in numerous preclinical experimental studies (eg, Coe et al, 2003; Kreider et al, 2006; Ladd et al, 2000; Maccari et al, 2003; Macri et al, 2007; Owen et al, 2005; Weaver et al, 2001; Welberg and Seckl, 2001). Early-life stress (ELS) at moments when critical developmental processes are taking place in parts of the nervous system or neuronal circuits involved in (later) HPA-axis functioning, may induce epigenetic changes that program the HPAaxis. This possibly results in some individuals in distinct and stable patterns of dysregulations that are associated with altered emotional processing and heightened responsiveness to stress (Heim et al, 2004; Meaney and Szyf, 2005; 
Weaver et al, 2001, 2005). In humans, the effects of ELS were tested in epidemiological studies (see Kajantie, 2006; Phillips, 2004; Thompson et al, 2001) and in retrospective (see Heim et al, 2000, 2004; Gunnar and Quevedo, 2007; Kaufman and Charney, 2001; Tarullo and Gunnar 2006) and prospective (see Davis et al, 2005; Egliston et al, 2007; Huizink et al, 2004; Talge et al, 2007; Van den Bergh et al, 2005a) clinical and quasi-experimental studies.

Some prospective studies established the nature and timing of ELS exposure in relation to HPA-dysregulation in $5 \frac{1}{2}$ year olds (Gütteling et al, 2005), in prepubertal (Young et al, 2006), 10-year-old (O'Connor et al, 2005), and 13year-old children (Halligan et al, 2004). To the best of our knowledge, only one prospective study has established, via mediation analysis, the potential involvement of the HPAaxis in the link between ELS (namely maternal postnatal depression) and depressive symptomatology in 16-year-old postpubertal adolescents (Halligan et al, 2006). The present study builds on this work in extending the early-life indices to the prenatal period with the use of antenatal maternal anxiety measures.

Studies of the past 35 years have revealed that MDD is characterized by a hyperactive HPA-axis induced by alteration at different levels of the HPA-axis. Corticotropinreleasing hormone (CRH)-hyperdrive (at hypothalamic and extrahypothalamic levels), probably potentiated by the action of arginin vasopressin, as well as a reduced negative feedback have been proposed as the primary factor in the pathogenesis of MDD that contribute to the observed elevation of cortisol in MDD. However, the exact pathophysiology of MDD, which also involves changes in monoamine neurotransmitters and the immune system, remains to be unraveled further (see Claes, 2004; Claes and Nemeroff, 2005; Chrousos, 1998; Heim et al, 2004; Holsboer, 2001; Pruessner et al, 2003; Raison and Miller, 2003; Swaab et al, 2006; van Praag et al, 2004; Van Den Eede et al, 2005; Van West et al, 2006).

The secretion of cortisol, the hormonal endproduct of HPA-axis activation, is kept within an optimal timeintegrated narrow range, which is quite stable in an individual subject (Chrousos et al, 2004). Normally, basal cortisol increases markedly upon awakening to peak at 30-40 min post-awakening, followed by a declining pattern until the evening and a quiescent period (a dip) with very low cortisol around 2000-0200 hours (van Praag et al, 2004), and finally a rise into the next awakening surge. Changes in or dysregulation of the diurnal rhythm of cortisol are increasingly being studied in depression and other stress-related disorders in which the HPA-axis is involved (Chrousos and Gold, 1998; den Hartog et al, 2003; Deuschle et al, 1997; Gunnar and Vazquez, 2001; Heim et al, 2000; Holsboer et al, 1984; Miller et al, 2007; Rosmond et al, 1998; Sachar et al, 1970). A high, flattened diurnal profile has been shown to be indicative of a chronically stressed, hyperactive HPA-axis (Deuschle et al, 1997; McEwen, 2002: McBruce and Wingfield, 2003; Rosmond et al, 1998) and has been observed in depressed adult (den Hartog et al, 2003; Sachar et al, 1970; Young et al, 2006) and adolescent (Kaufman and Charney, 2001; Forbes et al, 2006) outpatients. Such a profile has moderately lower awakening output with less decline in the afternoon/evening than would be expected, thus leading to a higher daily output.
Other dysregulations imply low cortisol levels at awakening which remain constant throughout the day. Such a low, flattened diurnal profile (ie, lacking of expected diurnal rhythm) reflects reduced cortisol output (hypocortisolism) and is seen in adult patients with post-traumatic stress disorders, atypical depression, or chronic fatigue syndrome (Gunnar and Quevedo, 2007; Heim et al, 2000, 2004; Miller et al, 2007; Van Praag et al, 2004) and in neglected children (eg, orphanage-reared children; see Gunnar and Vazquez, 2001; Gunnar and Quevedo, 2007). Total cortisol secretion measures or single measures at one moment during the day do not take account of circadian or diurnal variability and may therefore be less sensitive markers of vulnerability than measures of diurnal or circadian cortisol rhythm (Chrousos and Gold, 1998). Therefore, this paper will focus on measures of cortisol rhythm.

In a recent meta-analysis, Miller et al (2007) concluded that even when a person does not develop a full-blown psychiatric condition, the extent of distress is positively associated with HPA-activation. To the extend that people reported higher levels of distress, they show greater daily cortisol output and afternoon/evening cortisol, although morning levels are somewhat lower. However, the major limitation of the work on which that conclusion is based is that is has been largely cross-sectional in nature. Miller et al (2007) accentuate that future work in this area needs to be prospective. Such work could identify psychopathological or 'predisease' pathways at the medium-low part of the continuum of depressive symptomatology, with the use of biological markers - vulnerability markers. This may lead to a significant progress in the early identification of vulnerable persons and to the elaboration of early intervention programs to delay or prevent diseases later in life (McEwen, 2002). Moreover, post-pubertal adolescents have been understudied with respect to diurnal cortisol rhythms. It is suggested that the full maturation of the HPA-axis - including the full diurnal cortisol rhythm - which is reached with the attainment of Tanner stage three, may have implications for the heightened risk of psychopathology noted among post-pubertal adolescents (Gunnar and Quevedo, 2007).

In the present study, we aimed to investigate the possible fetal origins of disturbance in diurnal cortisol rhythms and depressive symptomatology in post-pubertal adolescents. To do so, we tested the following two hypotheses in a sample of an ongoing prospective-longitudinal study started at 12-22 weeks of pregnancy (Van den Bergh, 1992, 2005b, 2006; Van den Bergh and Marcoen, 2004): (1) antenatal exposure to maternal anxiety is, in post-pubertal adolescents, associated with disturbance in the diurnal secretion pattern of cortisol; (2) disturbance in the diurnal secretion pattern of cortisol mediates the association between exposure to antenatal maternal anxiety and self-reported depressive symptoms in post-pubertal adolescents. Given the evidence outlined above, a disturbance in the diurnal cortisol profile could be reflected in a flattened profile. We also tested the effect of gender and of timing of anxiety and controlled for potential confounders. Significant results may be indicative that ELS during one or more critical gestation periods plays a role in the pathophysiology of depressive symptomatology and may provide preliminary evidence that 'programming' of the HPA-axis by exposure to antenatal maternal anxiety/stress may predispose individuals to depressive symptomatology even much later in life. 


\section{MATERIALS AND METHODS}

\section{Participants}

The study started with 86 healthy Dutch-speaking mothers (18-30-year-old) and their firstborn. Most of them were well educated and married and none of them had a psychiatric disorder. All babies were delivered between 36 and 41 weeks of pregnancy (wp) and had 5-min Apgar scores of 9 or 10 (2 babies with score 8 ). This sample has been assessed at 36-38 wp, at 1, 10, and 28 weeks after birth (Van den Bergh, 1992) and at ages 8-9 (Van den Bergh and Marcoen, 2004). At $14-15$ years of age, $68(79.1 \%)$ adolescents and their parents participated (Van den Bergh et al, 2005b, 2006); they all gave their informed consent. The ethical committee for experiments on human beings of our university approved the study.

\section{Scales Measuring Maternal Anxiety and Adolescent Depressive Symptomatology}

We used Dutch, psychometrically validated versions of standardized English symptom severity rating scales. The State Trait Anxiety Inventory (STAI) consists of a state and a trait subscale, each containing 20 items scored from 1 to 4 , and with reliability coefficients, Cronbach's alphas, of 0.95 and 0.93, respectively (Van der Ploeg et al, 1980). State anxiety is conceptualized as a transient emotional condition, whereas trait anxiety reflects a dispositional anxiety proneness. The mothers completed the STAI at all assessments; all data were used in the present study. The Children's Depression Inventory (CDI; Kovacs, 1992; Timbremont and Braet, 2002), which was completed by the adolescents, entails 27 items measuring the severity of depressive symptoms, scored from 0 to 2 . Subjects are asked to refer to their feelings, cognitions, and behavior during the past 2 weeks. According to the authors of the CDI, a score of 19 best distinguishes between nondepressed and clinically depressed persons rated with DSM-IV criteria, whereas levels between 13 and 18 are regarded as subclinical or minor depressive episode. In a Dutch-speaking reference population of 13-15-year-olds (Timbremont and Braet, 2002) the Cronbach's $\alpha$ is 0.84 and the test-retest reliability coefficient (Pearson product moment correlation) is 0.86 .

\section{HPA-Axis Dysregulation: Day-Time Cortisol Profile (Shortened Version)}

Saliva sampling, in the morning as well as during the day, is best done with strict reference to the timing of awakening rather than on exact, predefined hours; the latter underlies large inter-and intraindividual variation (Edwards et al, 2001; Pruessner et al, 1997, 2003). We used a short version of the day-time cortisol profile and samples were collected at awakening, at approximately 4 (noon) and $12 \mathrm{~h}$ (evening) after awakening, at home, on a typical weekend day. For reasons of feasibility, we planned the saliva collection on a weekend day (eg, for an adolescent on a normal school day it is difficult to collect the noon sample $4 \mathrm{~h}$ after the awakening sample). It is shown that there is not a significant impact of genetic factors on this day-time cortisol profile (Wüst et al, 2000). Considering our interest in prenatal environmental factors, a short day-time cortisol profile should thus be an appropriate measure.

Samples were collected by spitting in a small plastic tube (Sarstedt, Germany), without using aids to salivation or swabs. Detailed instructions were outlined on a sheet provided with the tubes. The tubes were kept refrigerated and brought along to the laboratory visit, together with the questionnaires they had completed at home. All samples were stored at $-60^{\circ} \mathrm{C}$ upon arrival. Cortisol in saliva was measured with a revised version of the protocol provided by the manufacturer of the Coat-a-Count Radio-ImmunoAssay Kit (Euro DPC, Llanberis, Wales). Two hundred microliters of saliva and diluted standards was used, with incubation for $3 \mathrm{~h}$ at room temperature, modified by an additional period overnight $(15-18 \mathrm{~h})$ at $+4^{\circ} \mathrm{C}$ (cf., O’Connor et al, 2005).

\section{Statistical Analyses}

The first hypothesis, on the relation between ELS and HPAaxis dysregulation, is tested using longitudinal repeated measurements regression. The outcomes were the three cortisol measurements that were encoded as 0 (awakening), 4 (noon), and 12 (evening). The associations among these measurements were best modeled using a 'heterogeneous first-order autoregressive' covariance structure. The independent ELS variables were the six prenatal maternal anxiety measures. Using six anxiety measures should bring about caution in the interpretation of the results, as we have six opportunities to find a relationship between prenatal anxiety and HPA-axis dysregulation. However, previous research has shown that fetal programming may occur during various gestational periods (for a review, see Talge et al, 2007; Van den Bergh et al, 2005a). It is supposed that the final programming effects are related to the kind of critical developmental processes that were taking place in organs or biological systems involved, at the time of ELS exposure. Hence, it is important to investigate the effect of exposure to maternal anxiety at different pregnancy periods. Repeated measurements allow the investigation of the whole cortisol profile and can thus be used to investigate the potential importance of a flattened profile. As an additional analysis, we also investigated the effect of prenatal anxiety on often-used summary measures of cortisol such as area under the curve (AUC). Note that these measures summarize the cortisol profile and as such loose a good deal of information.

The second hypothesis, on the mediational role of the HPA-axis between ELS and adolescent depression, was tested by a mediation analysis consisting of three steps, as required by the classical approach (Baron and Kenny, 1986). Using ordinary least-squares (OLS) regression, we assessed the influence of: (1) antenatal maternal anxiety on HPA-axis function (ie, cortisol day-time profile); (2) antenatal maternal anxiety on adolescent depressed mood; and (3) HPA-axis function on adolescent depressed mood when statistically controlling for antenatal anxiety. If the effect of antenatal anxiety on adolescent depressed mood (second regression) is less strong when HPA-axis function is also added to the model (third regression) and antenatal anxiety is associated with HPA-axis function (first regression), altered HPA-axis function can be seen as a mediator of the 
effect of antenatal anxiety on depressed mood. Interaction effects with gender were always added to the regressions to investigate the moderating effects of gender.

As an alternative to the classical approach to detect mediation, the recently suggested and highly powerful biascorrected bootstrap method (MacKinnon et al, 2004) was also used. The mediation effect is quantified by multiplying the parameter estimate of the antenatal maternal anxiety effect in the first regression with the parameter estimate of the HPA-axis function effect in the third regression. A $95 \%$ confidence interval of this mediation effect estimate was computed using the bias-corrected bootstrap method (MacKinnon et al, 2004).

Apart from gender, five covariates were considered: (a) smoking during pregnancy (binary); (b) birth weight controlled for gestational length (continuous); (c) obstetric optimality (continuous; a list of 52 optimal obstetrical conditions); (d) postnatal maternal trait anxiety (continuous: $z$-score resulting from a principal component analysis on trait anxiety measures at 1, 10, 28 weeks, $8 / 9$ and $14 / 15$ years after birth); and (e) morphological pubertal stage (ordinal: schematic drawings of secondary gender characteristics; according to Tanner, 1962, stage $\geqslant$ III indicates post-puberty).

Due to the small sample size and the complexity of the statistical analyses, the influence of different prenatal anxiety measures in the repeated measurements analysis and of the covariates in the mediation analyses were checked using model selection procedures. As a measure of effect size in OLS regression, the omega squared $\left(\omega^{2}\right)$ was computed; it represents the proportion of the variance of the outcome variable that is accounted for by a predictor variable. Based on the studentized residuals (absolute value $>2.00$ ), we defined cases as suspect (possible outliers). Removal of those cases did not change the conclusions, therefore they were retained in the analyses.

\section{RESULTS}

\section{Preliminary Statistics}

Of the 68 mother-child pairs, 58 (85.3\%) had complete data for all cortisol measures (29 males, 29 females; mean age $\pm \mathrm{SD}=15$ years 0 month \pm 3 months; range $=14$ years 6 months to 15 years 6 months). For the 58 complete cases, descriptive statistics are given in Table 1. State and Trait anxiety scores in the highest quartile correspond with deciles 6-9 (for anxiety at 23-31 and at 32-40 wp) and 6-10 (for anxiety at 12-22 wp) of a nonclinical female community sample (Van der Ploeg et al, 1980), indicating that for $25 \%$ of the pregnant women anxiety levels were at least higher than average.

Table I Descriptive Statistics for Key Variables $(n=58)$

\begin{tabular}{|c|c|c|c|c|}
\hline Variable & Mean (SD) & Median & QI-Q3 & Min-Max \\
\hline \multicolumn{5}{|l|}{ Maternal anxiety 12-22wp } \\
\hline Trait anxiety 12-22 wp & $36.0(8.44)$ & 35.5 & $29-39.5$ & $21-59$ \\
\hline State anxiety $12-22 \mathrm{wp}$ & $39.4(8.83)$ & 37.0 & $33-44$ & $24-62$ \\
\hline TAlI2-22 & $0.0(6.5 \mathrm{I})$ & 0.2 & $-4.0-4.1$ & $-16.0-14.7$ \\
\hline Trait anxiety $23-31 \mathrm{wp}$ & $35.0(8.25)$ & 34.5 & $28.0-41.0$ & $21.0-52.4$ \\
\hline State anxiety $32-40 w p$ & $35.8(7.21)$ & 34.7 & $30.0-40.0$ & $20.0-58.0$ \\
\hline \multicolumn{5}{|l|}{ Cortisol (nmol/l) } \\
\hline Awakening cortisol level & $12.3(4.99)$ & 11.8 & $9-15.4$ & 1.5-25.4 \\
\hline DIF (males) & I0.8 (3.38) & 9.9 & $9.0-13.3$ & $3.9-16.7$ \\
\hline \multicolumn{5}{|l|}{ Adolescent depressive symptoms } \\
\hline Children's depression inventory & $8.9(5.44)$ & 7.5 & $5-12$ & $1-25$ \\
\hline \multicolumn{5}{|l|}{ Gender and covariates } \\
\hline Adjusted birth weight & $-0.011(0.964)$ & -0.045 & $-0.76-0.90$ & $-1.85-1.88$ \\
\hline Obstetric optimality & $45.5(3.34)$ & 46.0 & $44-48$ & $30-51$ \\
\hline Postnatal maternal anxiety & $0.013(1.032)$ & -0.055 & $-0.72-0.81$ & $-2.12-2.34$ \\
\hline Pubertal development & $4.13(0.053)$ & 4.00 & $4-4.5$ & $3-5$ \\
\hline
\end{tabular}




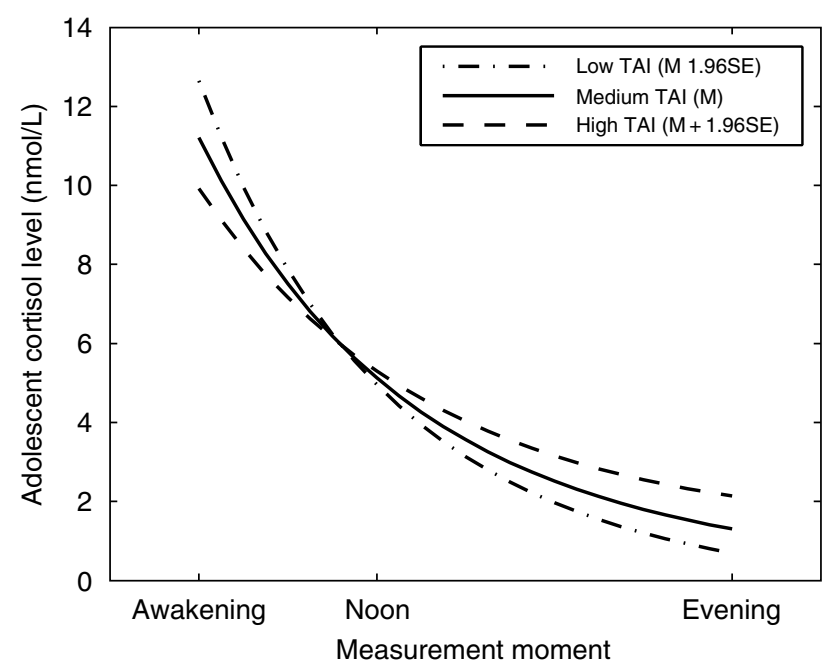

Figure I Day-time cortisol profiles as predicted by prenatal anxiety (TAII2-22) in a repeated measurements regression analysis.

As expected, the intraindividual variation in cortisol levels is large (see Table 1). The mean absolute levels of cortisol secretion at awakening, noon, and evening and the day-time profile in our sample (see also Figure 1) are in accordance with community samples with depressive outpatients and controls (eg, Peeters et al, 2004; Young et al, 2006).

The whole range of depressive symptomatology measured by the CDI is covered by our sample. If we use the cut-off scores defined by the authors of the CDI, $22 \%$ ( $n=13$; eight girls, five boys) of our sample can be seen as reporting at least subclinical depression symptoms (38\% of which had a CDI score higher than 18 ( $n=5$; four girls, one boy). An even higher percentage may suffer from depressed mood. This incidence seems comparable with the incidence in nonclinical adolescent samples, in which the likelihood of depressed mood ranges between $25-40 \%$ (girls) and $20-35 \%$ (boys) (Hankin and Abramson, 2001).

To correct for non-normality of the depressive symptoms scores and the cortisol values, a square-root, respectively $\log$ transformation was used for these variables.

\section{Repeated Measurements Analysis}

The effects considered were all six prenatal anxiety main effects, the linear, and quadratic time effects of cortisol, and the interactions of all six prenatal anxiety measures with the linear time effect (14 effects). After model selection, the model contained the linear and quadratic time effects of cortisol and all main and interaction effects related to anxiety at 12-22 wp (six effects). There was a linear and a quadratic time effect $(P<0.0001$, respectively $P=0.0363)$, suggesting that the cortisol level strongly decreased towards the evening and that this decrease was very strong at first, but less strong in the end, when approaching the quiescent period. Trait anxiety at $12-22 \mathrm{wp}$ emerged as the only prenatal anxiety predictor of the offspring day-time cortisol profile (interaction with the linear time effect: $\left.b \pm \mathrm{SE}=0.0027 \pm .0014, t_{(112)}=2.00, P=0.0476\right)$. Although surprising, the prenatal trait anxiety measures did not appear stable. The 12-22 wp period seemed especially influential for the mothers. Although traits are considered stable entities over short time spans, the trait anxiety in the 12-22 wp period tended to differ nonetheless from the two subsequent prenatal measures (Wilcoxon signed rank test: $S=216.5, P=0.09)$. This lack of stability may explain why the three trait measures do produce different results in our analysis.

The interaction between trait anxiety $12-22 \mathrm{wp}$ and the linear time effect suggested that adolescents whose mothers were anxious during 12-22 wp have a flattened day-time cortisol profile. It was also observed that state anxiety acted as a suppressor of trait anxiety. (Suppression is the counterintuitive situation where, in its classical form, a suppressor variable $\mathrm{X} 2$ is unrelated to outcome $\mathrm{Y}$ even though its inclusion in the model improves the prediction by making variable X1 an improved predictor of Y. In such a situation, $\mathrm{X} 2$ is correlated with a part of $\mathrm{X} 1$ that is uncorrelated with $\mathrm{Y}$ such that the addition of X2 to the model removes or suppresses this noise or unwanted variance in $\mathrm{X} 1$, thus enhancing the predictive ability of X1. Readers are referred to the seminal textbook by Cohen and Cohen (1983) and to the recent paper by Friedman and Wall (2005)). Therefore, we decided to control for state anxiety at $12-22 \mathrm{wp}$ in further analyses concerning trait anxiety at $12-22 \mathrm{wp}$. A parsimonious way to do so is to take the residuals of trait anxiety when it is regressed on state anxiety; this allows us to avoid the suppressing effect of state anxiety without having to include both variables in analyses. This variable is named 'trait anxiety index 12-22 wp' (TAI12-22). The correlation between trait anxiety at $12-22$ wp and TAI12-22 is 0.77 .

Higher TAI12-22 predicted a flattened day-time cortisol profile (ie, a smaller decrease from awakening cortisol to evening cortisol; see Figure 1). Also, the weak main effect of TAI12-22 indicated that it appears to be the absolute decrease in cortisol that is most important. These results suggested that the difference between the awakening and evening cortisol level - a measure named 'DIF' - was a valid proxy for the short day-time cortisol profile. DIF is a parsimonious measure to indicate the flatness of the cortisol profile; it has been used in previous research (Wüst $e t a l$, 2000). As the third regression in the mediation analysis uses the HPA-axis function as a predictor variable, DIF is interesting because it allows us to summarize the cortisol profile with one number reflecting that part of the profile, which is related to maternal anxiety. A profile in which awakening output is only somewhat lower, but in which there is less decline in the afternoon/evening than would be expected, results in a high, flattened profile, whereas a profile with low cortisol levels at awakening which remain constant throughout the day, results in a low, flat profile. Only in the latter case DIF will be very small, almost reaching zero.

We did additional analyses to check whether our results are related to a high flat profile or to a low flat profile. First, we redid all analyses, whereas excluding the four cases with the lowest awakening cortisol (which all had a low, flat profile and a low DIF). All results were similar and the conclusions were the same as with analyses including these four cases. Second, we compared adolescents whose mothers were not very anxious $(25 \%$ mothers with lowest $\mathrm{TAI} ;<\mathrm{Q} 1)$ and those that were highly anxious $(25 \%$ 
mothers with highest TAI; >Q3). We observed that the relation between TAI and DIF was not due to cases with low awakening cortisol; these cases typically had mothers with an average TAI (ie between Q1 and Q3). Adolescents of highly anxious $v s$ low anxious mothers had the following median cortisol levels: 13.3 at awakening (mean $=12.7$ ), 5.9 at noon, 1.5 in the evening $v s 13.2$ at awakening $($ mean $=14.4), 5.0$ at noon, 0.4 at evening.

Using OLS regression analysis, no strong effects of antenatal maternal anxiety on the cortisol awakening or AUC measures were found (all $P>0.21$ ).

\section{Mediation Analyses}

The first regression conceptually replicated the results of the repeated measurements analyses. The TAI12-22 anxiety index is used to predict the diurnal cortisol change (DIF). No effects involving gender or the covariates were important. TAI12-22 was associated with lower DIF, thus with less decreasing (ie, flattened) cortisol profiles $\left(b \pm S E=-0.210 \pm 0.103, t_{56}=-2.03, P=0.0467, \omega^{2}=0.05\right)$. Obviously, this result is nearly identical to the interaction effect described in the previous section.

In the second regression, the gender-TAI12-22 interaction $\left(b \pm S E=0.083 \pm 0.035, \quad t_{54}=2.36, \quad P=0.0220, \quad \omega^{2}=0.07\right.$; Figure 2) revealed that higher TAI12-22 predicts higher depressed mood only in adolescent females (females: $P=0.0027, \omega^{2}=0.14$; males: $P=0.8470, \omega^{2}=0.00$ ). Including the other covariates only yielded a significant positive effect of postnatal maternal anxiety. This effect did not eliminate the gender/TAI12-22 interaction $(P=0.0502$, $\left.\omega^{2}=0.04\right)$.

In the third regression, postnatal maternal anxiety was again the only covariate retained in the model. A gender-DIF interaction $\left(b \pm S E=-0.096 \pm 0.048, t_{51}=-2.02\right.$, $P=0.0492, \omega^{2}=0.04$; Figure 3 ) showed that smaller DIF (ie, a flattened profile) predicts higher depressed mood, again in females only (females: $P=0.0077, \omega^{2}=0.08$; males: $\left.P=0.4216, \omega^{2}=0.00\right)$. Importantly, the strength of the gender-TAI12-22 interaction was smaller than in the second

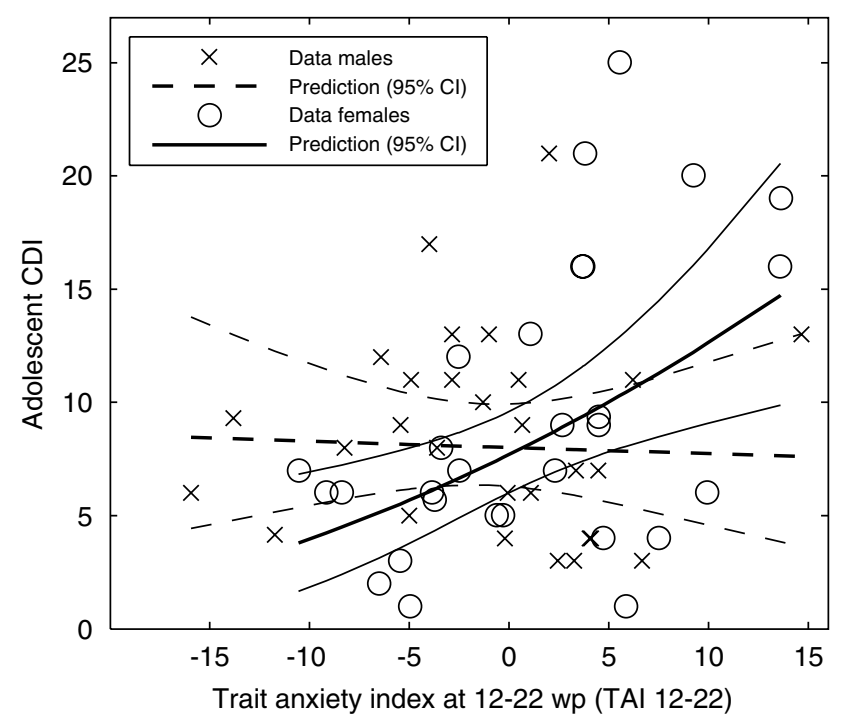

Figure $2 \mathrm{CDI}$ depressive symptoms scores as predicted by prenatal maternal anxiety (TAlI2-22) and gender in the second regression. regression $\left(P=0.1735, \omega^{2}=0.01\right)$. This indicates that, only in females, there is an effect of antenatal exposure to maternal anxiety on depressed mood in adolescence that can be explained, in part, by an effect of the flattened cortisol profile on depressed mood (this is mediation according to the classical approach) (Figure 4, panel b).

Regression analyses in which cortisol or depressed mood were regressed on measures of anxiety at 23-31 and 32-40 wp did not reveal independent effects of anxiety during these periods of pregnancy.

The alternative bias-corrected bootstrap method (MacKinnon et al, 2004) to investigate mediation also suggested a mediating role of DIF for girls. Because the gender-dependent effect of TAI12-22 on CDI is at least partially mediated by a general effect of TAI12-22 on DIF and a gender-dependent effect of DIF on CDI, the mediation effect could be quantified by multiplying the parameter estimates of the effect of TAI12-22 on DIF (first regression)

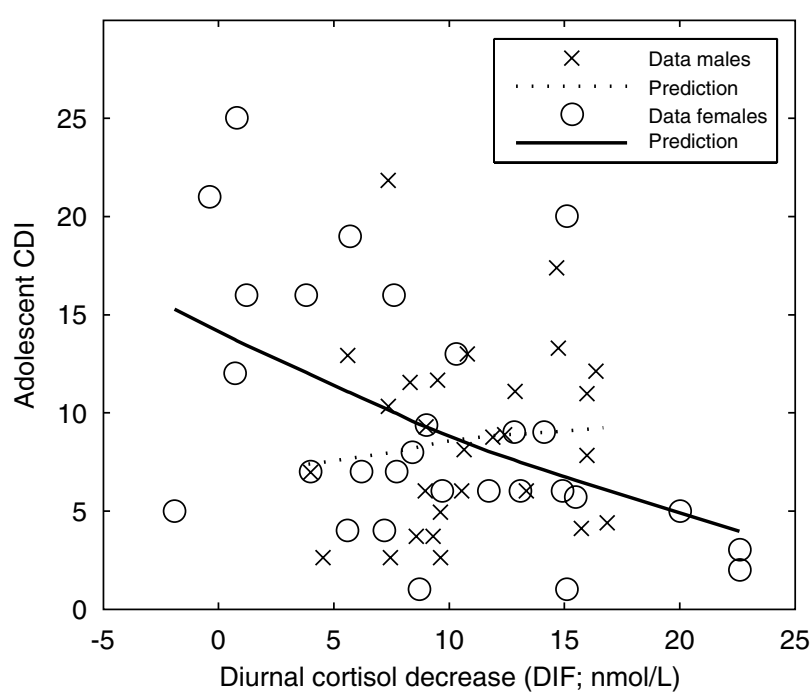

Figure $3 \mathrm{CDI}$ depressive symptoms scores as predicted by diurnal cortisol decrease (DIF) and gender in the third regression. The prediction lines are smoothed to filter out the influence of other variables.

a

Hypothesis 1 (confirmed in both genders)

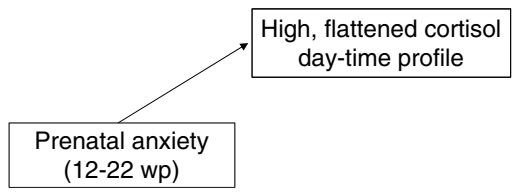

b Hypothesis 2 (only confirmed in females)

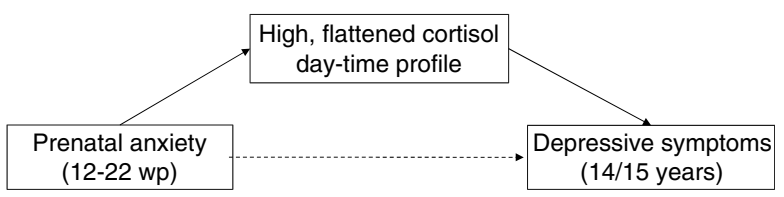

Figure 4 Graphical presentation of the results. Results in (a) represent the effect of prenatal anxiety on a flattened day-time cortisol profile. Results in (b) represent a 'mediated moderation' model in which a gender-related effect of prenatal anxiety on adolescent depressive symptoms (dashed line) is mediated by a flattened day-time cortisol profile. 
and the gender-dependent effect of DIF on CDI: -0.021 $*-0.096=0.0202$. The $95 \%$ confidence interval of this mediation effect equaled $(0.0025 ; 0.0603)$, suggesting the presence of mediation.

\section{DISCUSSION}

This study prospectively investigated the influence of antenatal maternal anxiety on HPA-axis vulnerability and self-reported depressive symptoms in 58 14- and 15-yearolds. Our results showed that anxiety at 12-22 weeks of pregnancy was associated with a diurnal cortisol profile that was attenuated due to somewhat lower than normal cortisol output at awakening, but higher than expected secretion in the evening, in females as well as in males. Maternal anxiety at 23-31 and 32-40 weeks of pregnancy had no independent effect on diurnal cortisol profile. Furthermore, in female offspring only, the flattened profile was shown to mediate the link between maternal anxiety at 12-22 wp and depressive symptoms. These results were found after controlling for obstetric risk, postnatal maternal anxiety, and other potential confounders. These results confirm our two hypotheses and furthermore reveal an effect of gender (see Figure 4).

We have obtained several interesting findings, which of course need replication in larger samples before firm conclusions can be drawn.

First, in line with previous results of preclinical, retrospective, and prospective ELS studies, we find that exposure to adverse environmental cues during presumed critical periods of time in early life influence later vulnerability and health. We can conclude that our empirical evidence is consistent with the 'fetal programming'-hypothesis, which more recently has also been labeled 'developmental origins of health and disease (DOHaD) - hypothesis' (eg, Gluckman and Hanson, 2004).

Second, to our knowledge this is the first prospective study that tentatively illustrates the potential involvement of the HPA-axis in the link between antenatal maternal anxiety (or prenatal ELS) and depressive symptoms in postpubertal, female adolescents. These results can be seen as an extension of the findings of Halligan et al (2006) up into the prenatal life period.

Although we cannot proof it, 'resetting of the HPA-axis setpoints' by antenatal exposure to maternal anxiety during critical periods leading to a hyperactive HPA-axis, seems a plausible underlying mechanism (cf., Levine, 2005; Weaver et al, 2001; Owen et al, 2005). A high, flattened diurnal profile has in other studies been empirically linked with inadequate suppression of awakening cortisol by overnight dexamethasone, for example, in chronically distressed (Rosmond et al, 1998) and in depressed adults (Young et al, 2006). According to Chrousos and Gold (1998), the findings of Rosmond et al (1998) suggest chronic hypersecretion of CRH and a reset of their HPA-axis. Earlier results of Deuschle et al (1997) (24-h blood sampling) lend support to this kind of interpretation of a high, flattened profile. If this interpretation holds also for the high flattened profile following prenatal ELS in our sample, our results may also be seen as a possible extension into the prenatal life period of the finding of Heim et al (2004) that CHR-overdrive is observed in adults who were maltreated as a child, and particularly, among adult survivors of maltreatment who have depression. Some studies have found that early postnatal ELS is associated with a low, flat cortisol profile (for a review, see Gunnar and Vazquez, 2001; Heim et al, 2000; Tarullo and Gunnar, 2006). Notwithstanding the fact that some adolescents had a low flat cortisol profile (ie, those with a very low DIF; see Table 1), this profile was not associated with prenatal ELS (ie, exposure to maternal anxiety) in our study.

Third, with no known biological markers available to diagnose mood and anxiety disorders at an early stage, researchers previously had to rely solely on the use of behavioral signs and other nonbiological risk and resiliency factors in the prediction (eg, Ong et al, 2006) or prevention of depression (eg, Beardslee and Gladstone, 2001). Our results seem to indicate that, at least in female offspring of highly anxious pregnant women, a high and flattened diurnal profile of cortisol secretion may be seen as a measure of 'predisease' pathway (McEwen, 2002) or as a vulnerability marker (Gottesman and Gould, 2003).

Fourth, although our conclusion concerning the gender effect may be limited by the small number of males with depressive symptoms, a plausible interpretation is that maternal anxiety induced gender-specific changes in the HPA-axis during critical gestation periods, which become clear in post-puberty, when the HPA-axis has reached its full maturation. This may render females more vulnerable: more sustained HPA-axis activation, a lower threshold for perceiving stress, increased vigilance, and sensitization of emotional and HPA-axis responses to subsequent stress may have enhanced the risk to develop depressive symptoms in females. Of course, it is also plausible that only during puberty females become more vulnerable to depressive symptoms because of crucial gender differences in gonadal hormones (Ge et al, 2006; Netherton et al, 2004; Kessler, 2003; Federman 2006; Goodyer et al, 2000; Kajantie and Phillips, 2005).

Fifth, in our sample, we did not have evidence of traumatic experiences such as maltreatment that may have interfered with the effects of intrauterine life. Results of this wave and of earlier waves of our longitudinal study are consistent with the idea of 'fetal programming', namely that antenatal maternal anxiety already before birth may have altered the neurodevelopmental program of the offspring. Maternal anxiety in early-mid (12-22 wp) as well as in late pregnancy (23-31 and 32-40 wp) were associated with fetal sleep and movements patterns observed at 36-38 weeks (Van den Bergh, 1992) and moreover, at $8 / 9$ years $15 \%$ of anxiety symptoms, measured with a child-report standardized anxiety scale, were explained by prenatal maternal state anxiety at 12-22 wp (Van den Bergh and Marcoen, 2004). Enhanced anxiety in the child may reflect sensitization of the HPA-axis and may be seen as co-morbid symptoms (Halbreich, 2006) or a precursor of later depressive symptoms (Forbes et al, 2006).

Sixth, we observed that, in our sample, different periods exist during which the fetus is susceptible for programming. Effects of maternal anxiety on childhood disorders at 8-9 years, on cognitive functioning (Van den Bergh et al, 2005b, 2006) and on depressive mood at 14-15 years are confined to maternal anxiety at $12-22 \mathrm{wp}$, whereas those on fetal 
sleep and movement patterns were not. This is consistent with the idea that developmental programming may operate at least during the whole prenatal period and that timing effects of ELS exposure may depend on the timing of critical developmental processes in underlying neural circuits and other biological system involved. In this regard, it is interesting that several gestational periods were reported to be critical for the development of ADHD in childhood (for a review, see O'Connor et al, 2003; Rodriguez and Bohlin, 2005; Van den Bergh and Marcoen, 2004); this may indicate that various brain systems, that each have different critical periods, are underlying different types of ADHD.

The limitations of our study necessitate some caution in interpreting its results and their implications. First, the sample was rather small and results might be sample specific; confirmation on larger samples is required. Second, our HPA-axis measures showed some shortcomings. Assessment of maternal HPA-axis during pregnancy and of HPAdysregulation in the offspring at an earlier age would have enabled us to better test their assumed role in the chain of pathophysiological events. As it is known that saliva cortisol shows day-to-day variability, assessment of salivary cortisol on more than one day could have increased the data reliability (Pruessner et al, 1997; Wüst et al, 2000). We planned the saliva collection on a weekend day for reasons of feasibility. Adolescents sleep in on weekend days. Adolescents with depressed mood may sleep in more than adolescents without depressed mood. It can be questioned whether that may explain why adolescents with a depressed mood show a flatter cortisol profile. We do not think this is a plausible explanation for our data; whereas sleeping in on weekend day should mainly be related to lowering of the awakening cortisol, the flattening of the daytime profile was mainly a consequence of the elevated evening cortisol and not of lower awakening cortisol. Also, although preclinical studies (Maccari et al, 2003) and previous ELS studies (Heim et al, 2000; Gunnar and Quevedo, 2007) have observed that reactivity to a stressor may be a more sensitive measure of HPA-axis functioning than diurnal salivary cortisol, we were not able to include this measure in our study. Finally, we did not measure the adolescents' stress concurrently with their cortisol assessment, therefore, we cannot rule out its influence on cortisol (Goodyer et al, 2000).

Third, as the HPA-axis is connected with other biological systems (eg, monoamine neurotransmitters, immune system), which we did not measure, it would be premature to conclude that the HPA-axis is the only system involved in linking ELS to depressive symptoms (cf., O'Connor et al, 2005).

Fourth, our study has no genetic sensitive design, therefore, we cannot exclude an effect of genes or a geneenvironment interaction nor can we explore the interesting hypothesis that prenatal adversity may 'amplify' eventually existing genetic differences (de Kloet et al, 2005).

To our defence, however, our study also has some strengths compared to earlier work in this area. First, it is based on a prospective design with a retention rate of 67 $(n=58)-79 \%(n=68)$ over 15 years. Second, we have used well-validated questionnaires and, third, our sample covered the range of maternal anxiety and adolescent depressive symptoms scores seen in nonclinical populations and a substantial proportion scored in the higher range. Fourth, rater bias was almost excluded as a confounding factor, because the mother rated her anxiety and the adolescent his/her depressive symptoms. Fifth, we have used a solid mediation analysis in which also the moderating effect of gender was tested and obstetric risk, postnatal anxiety and other confounding factors were controlled.

In conclusion, our findings may suggest that 'developmental programming' of the HPA-axis by maternal anxiety in early-mid pregnancy leads to vulnerability, predisposing females to develop depressive symptoms after puberty, and probably earlier. Specifically, we have argued for a hyperactive HPA-axis as a putative neurobiological mechanism leading to an enhanced risk for depression. Although it is clear that our results cannot directly prove this programming of HPA-axis hyperactivity and surely need to be replicated before firm conclusions can be drawn, they corroborate results from numerous preclinical and several human ELS studies and as such underscore the growing need to consider not only postnatal ELS, but also early prenatal ELS in any (single or combined) model of HPAaxis; vulnerability; depressed mood and depression (Gunnar and Quevedo, 2007; Heim et al, 2004). Undoubtedly, ELS has complex, long-term and gender-specific influences on health and disease. To be able to optimize strategies for prevention and treatment, studies should pay more attention to the nature, duration and timing of pre- and postnatal ELS, to potential vulnerability markers and to the identification of possible genetic and nongenetic factors (including diverse therapeutic interventions) that may enhance or protect, buffer or delay the risk ELS bears for later health.

\section{ACKNOWLEDGEMENTS}

We are extremely grateful to all families who took part in this study, to Vivette Glover, perinatal psychobiologist, who gave advice in collecting the saliva samples and had the samples analyzed in her lab, to Johan Wagemans, for useful comments on the previous draft of the manuscript, experimental psychologist to the psychologists who collected the data (Veerle Stevens, Tanja Geerdens) entered the data (Ann Theunissen) and searched relevant literature (Sylvia Pinna Puissant). This research is supported by Grant no G.0211.03 of the Fund for Scientific Research-Flanders (Belgium), by IUAP P06/04 (DYSCO) GOA-AMBioRICS, EU projects BIOPATTERN (FP6-2002-IST 508803) and eTUMOUR (FP6-2002-LIFESCIHEALTH 503094) and by grants IMPH/06/GHW and IDO 05/010 EEG-fMRI of the Katholieke Universiteit Leuven (KU Leuven).

\section{DISCLOSURE/CONFLICT OF INTEREST}

None of the authors has a potential conflict of interest. None of them has received a compensation for professional services in any of the previous three years, or anticipates receiving such compensation in the near future.

\section{REFERENCES}

Angold A, Costello EJ, Worthman CM (1998). Puberty and depression: the role of age, pubertal status and pubertal timing. Psychol Med 28: 51-61.

Baron RM, Kenny DA (1986). The moderator-mediator variable distinction in social psychological research: Conceptual, 
strategic, and statistical considerations. J Pers Soc Psychol 51: 1173-1182.

Beardslee WR, Gladstone TRG (2001). Prevention of childhood depression: recent findings and future prospects. Biol Psychiat 49: 1101-1110.

Claes SJ (2004). CRH, stress, and major depression: a psychobiological interplay. Vitam Horm 69: 117-150.

Claes SJ, Nemeroff CB (2005). Corticotrophin-releasing factor (CFR) and major depression: towards an integration of psychology and neurobiology in depression research. In: Corveleyn J, Luyten P, Blatt SJ (eds). The Theory and Treatment of Depression. Towards a Dynamic Interactionism Model. University Press: Leuven, Belgium and Erlbaum, Mahwah, NJ. pp 227-252.

Coe CL, Kramer M, Czeh B, Gould E, Reeves AJ, Krischbaum C et al (2003). Prenatal stress diminishes neurogenesis in the dentate gyrus of juvenile rhesus monkeys. Biol Psychiatry 54: 1025-1034

Cohen J, Cohen P (1983). Applied Multiple Regression/Correlation Analysis for the Behavioral Sciences, 2nd edn. Erlbaum: Hillsdale.

Chrousos GP (1998). Stressors, stress and the neuroendocrine integration of the adaptive response. Ann NY Acad Sci 851: 311-335.

Chrousos GP, Charmandari E, Kino T (2004). Editorial: Glucorticoid action networks - an introduction to systems biology. J Clin Endocrinol Metabol 89: 563-564.

Chrousos GP, Gold PW (1998). Editorial: A health body in a healthy mind-and vice versa-the damaging power of 'uncontrollable stress'. J Clin Endocrinol Metabol 56: 1842-1845.

Davis EP, Glynn LM, Dunkel Schetter C, Hobel C, Chicz-Demet A, Sandma CA (2005). Corticotropopin-relasing hormone during pregnancy is associated with infant temperament. Dev Neurosci 27: 299-305.

de Kloet ER, Sibug RM, Helmerhorst FM, Schmidt M (2005). Stress, genes and the mechanism of programming the brain for later life. Neurosci Biobehav Rev 29: 271-282.

den Hartog HM, Nicolson NA, Derix MMA, van Bemmel AL, Kremer B, Dolles J (2003). Salivary cortisol patterns and cognitive speed in major depression: a comparison with allergic rhinitis and healthy control subjects. Biol Psychiatry 63: 1-14.

Deuschle M, Schweiger UL, Weber B, Gotthardt U, Körner A, Schmider J et al (1997). Diurnal activity and pulsatility of the hypothalamus-pituitary-adrenal system in male depressed patients and healthy controls. J Clin Endocrinol Metabol 82: 234-238.

Edwards S, Clow A, Evans P, Hucklebridge F (2001). Exploration of the awakening cortisol response in relation to diurnal cortisol secretory activity. Life Sci 68: 2093-2103.

Egliston K-A, McMahon C, Austin M-A (2007). Stress in pregnancy and infant HPA axis function: conceptual and methodological issues relating to the use of salivary cortisol as an outcome measure. Psychoneuroendocrinology 32: 1-13.

Federman DD (2006). The biology of human sex differences. N Eng J Med 354: 1507-1514.

Forbes EE, Williamson DE, Ryan DN, Birmaher B, Axelson DA, Dahl RE (2006). Peri-sleep-onset cortisol levels in children and adolescents with affective disorders. Biol Psychiatry 59: 24-30.

Friedman L, Wall M (2005). Graphical views of suppression and multicollinearity in multiple linear regression. American Statistician 59: 127-136.

Ge X, Natsuaki MN, Conger RD (2006). Trajectories of depressive symptoms and stressful life events among male and female adolescents in divorced and nondivorced families. Dev Psychopathol 18: 253-274.

Gluckman PD, Hanson MA (2004). Living with the past: evolution, development and patterns of disease. Science 305: 1733-1736.

Goodyer IM, Herbert J, Tamplin A, Altham PME (2000). Recent life events, cortisol, dehydroepiandrosterone and the onset of major depression in high-risk adolescents. $\mathrm{Br} J$ Psychiatry 177: 499-504.
Gottesman I, Gould T (2003). The endophenotype concept in psychiatry: etymology and strategic intentions. Am J Psychiatry 160: $1-10$.

Gunnar MR, Vazquez DM (2001). Low cortisol and a flattening of expected daytime rhythm: potential indices of risk in human development. Dev Psychopathol 13: 515-538.

Gunnar M, Quevedo K (2007). The neurobiology of stress and development. Annu Rev Psychol 58: 145-173.

Gütteling BM, de Weerth C, Buitelaar JK (2005). Prenatal stress and children's cortisol reaction to the first day of school. Psychoneuroendocrinology 30: 541-549.

Halbreich U (2006). Major depression is not a diagnosis, it is a departure point to differential diagnosis-clinical and hormonal considerations. Psychoneuroendocrinology 31: 16-22.

Halligan SL, Herbert J, Goodyer IM, Murray L (2004). Exposure to postnatal depression predicts elevated cortisol in adolescent offspring. Biol Psychiatry 55: 376-381.

Halligan SL, Herbert J, Goodyer I, Murray L (2006). Disturbances in morning cortisol secretion in association with maternal postnatal depression predict subsequent depressive symptomatology in adolescents. Biol Psychiatry 60 (in press) doi: 10.1016/ j.biopsych.2006.09.011.

Hankin BL (2006). Adolescent depression: description, causes and intervention. Epilepsy Behav 8: 102-114.

Hankin BJ, Abramson LV (2001). Development of gender differences in depression: an elaborated cognitive vulnerability-transactional stress theory. Psycholog Bull 127: 773-796.

Heim C, Ehlert U, Hellhammer D (2000). The potential of hypocortisolism in the pathophysiology of stress-related bodily disorders. Psychoneuroendocrinology 25: 1-35.

Heim C, Plotsky PM, Nemeroff CB (2004). Importance of studying the contributions of early adverse experience to neurobiological findings in depression. Neuropsychopharmacology 29: 641-648.

Holsboer F (2001). Stress, hypercortisolism and corticosteroid receptors in depression: implications for therapy. J Affect Disord 62: 77-91.

Holsboer F, Bardeleben U, Gerken A, Stalla GK, Muller OA (1984). Blunted corticotropin and normal cortisol response to corticotropin-releasing-factor in depression. Engl J Med 311: 1127.

Huizink AC, Mulder EJH, Buitelaar JK (2004). Prenatal stress and risk for psychopathology: specific effects or induction of general susceptibility? Psychol Bull 130: 115-142.

Kajantie E (2006). Fetal origins of stress-related adult disease. Ann NY Acad Sci 1083: 11-27.

Kajantie E, Phillips DIW (2005). The effects of sex and hormonal status on the physiological response to acute psychosocial stress. Psychoneuroendocrinology 31: 151-178.

Kaufman J, Charney D (2001). Effects of early stress on brain structure and function: implications for understanding the relationship between child maltreatment and depression. Dev Psychopathol 13: 451-471.

Kessler RC (2003). Epidemiology of women and depression. J Affect Disorders 74: 5-13.

Kreider ML, Tata CA, Cousins MM, Olier CA, Seidler FJ, Slotkin TA (2006). Lasting effects of developmental dexamethasone treatment on neural cell number and size, synaptic activity and cell signaling: critical periods of vulnerability, dose-effect relationships, regional targets and sex selectivity. Neuropsychopharmacology 31: 12-35.

Kovacs M (1992). Children's Depression Inventory. Multi Health Systems: New York.

Ladd CO, Huot RL, Thrivikraman KV, Nemeroff CB, Meaney MJ, Plotsky PM (2000). Long-term behavioral and neuroendocrine adaptations to adverse early experiences. Progr Brain Res 122: 81-103.

Levine S (2005). Developmental determinants of sensitivity and resistance to stress. Psychoneuroendocrinology 30: 939-946. 
Maccari S, Darnaudery M, Morley-Fletcher S, Zuena AR, Cinque C, Van Reeth O (2003). Prenatal stress and long-term consequences: implications of glucocorticoid hormones. Neurosci Biobehav Rev 27: 119-127.

MacKinnon DP, Lockwood CM, Williams J (2004). Confidence limits for the indirect effect: distribution of the product and resampling methods. Multivar Behav Res 39: 99-128.

Macri S, Spinelli S, Adriani W, Highley JD, Laviola G (2007). Early adversity and alcohol availability persistently modify serotonin and hypothalamic-pituitary-adrenal-axis metabolism and related behavior: what experimental research on rodents and primates can tell us. Neurosci Biobehav Rev 31: 172-180.

McBruce BS, Wingfield JC (2003). The concept of allostasis in biology and biomedicine. Horm Behav 43: 2-15.

McEwen BS (2002). Sex, stress and the hippocampus: allostasis, allostatic load and the aging process. Neurobiol Aging 23: 921-939.

Meaney MJ, Szyf M (2005). Environmental programming of stress responses through DNA methylation: Life at the interface between dynamic environment and a fixed genome. Dialogues in Clinical Neuroscience 7: 103-123.

Netherton C, Goodyer J, Tamplin A, Herbert J (2004). Salivary cortisol and dehydroepiandrosterone in relation to puberty and gender. Psychoneuroendocrinology 29: 125-140.

Miller GE, Chen E, Zhou ES (2007). If it goes up, must it comd down? Chronic stress and thet hypothalamic-puitary-adrenocortical axis in humans. Psychol Bull 113: 25-45.

O'Connor TG, Ben-Shlomo Y, Heron J, Golding H, Adams D, Glover V (2005). Prenatal anxiety predicts individual differences in cortisol in pre-adolescent children. Biol Psychiatry 58: 211-217.

O'Connor TG, Heron J, Golding J, Glover V (2003). Maternal antenatal anxiety and behavioural/emotional problems in children: a test of a programming hypothesis. J Child Psychol Psychiatry 44: 1025-1036.

Ong SH, Wickramatne P, Tang M, Weissman MM (2006). Early childhood sleep and eating problems as predictors of adolescent mood and anxiety disorders. J Affect Disord 96: 1-8.

Owen D, Andrews MH, Matthews EJH (2005). Maternal adversity, glucocorticoids and programming of neuroendocrine function and behaviour. Neurosci Biobehav Rev 29: 209-226.

Peeters F, Nicolson N, Berkhof J (2004). Levels and variability of daily life cortisol secretion in major depression. Psychiatry Res 126: $1-13$.

Phillips DI (2004). Fetal programming of the neuroendocrine response to stress: links between low birth weight and the metabolic syndrome. Endocr Res 30: 819-826.

Pruessner JC, Wolf OT, Hellhammer DH, Buske-Kirschbaum A, von Auer K, Jobst S et al (1997). Free cortisol levels after awakening: a reliable biological marker for the assessment of adrenocortical activity. Life Sci 61: 2539-2549.

Pruessner M, Hellhammer DH, Pruessner JC, Lupien SJ (2003). Self-reported depressive symptoms and stress levels in healthy young men: associations with the cortisol response to awakening. Psychosom Med 65: 92-99.

Raison CL, Miller AH (2003). When not enough is too much: the role of insufficient glucocorticoid signaling in the pathophysiology of stress-related disorders. Am J Psychiatry 160: 1554-1565.

Rodriguez A, Bohlin G (2005). Are maternal smoking and stress during pregnancy related to ADHD symptoms in children. J Child Psychol Psychiatry 46: 246-254.

Rosmond R, Dallman MF, Björntorp P (1998). Stress-related cortisol secretion in men: relationships with abdominal obesity and endocrine, metabolic and hemodynamic abnormalities. $J$ Clin Endocrinol Metabol 83: 1853-1859.

Sachar E, Hellman L, Fukushima DK, Gallagher TE (1970). Cortisol productionin depressive illness. Arch Gen Psyhiatry 23: 289-298.

Swaab DW, Bao A-M, Lucassen PJ (2006). The stress system in the human brain in depression and neurodegeneration. Ageing Res Rev 4: 141-194.
Talge NM, Neal C, Glover V, and the Early Stress, Translational Research and Prevention Science Network (2007). Fetal and neonatal experience on child and adolescent mental health. Antenatal maternal stress and long-term effects on child neurodevelopment: how and why? J Child Psych Psychiatry 48: 245-261.

Tanner JM (1962). Growth at Adolescence: with a General Consideration of the Effects of Heridity and Environmental Factors upon Growth and Maturation from Birth to Maturity. Blackwell Scientific Publications: Oxford.

Tarullo AR, Gunnar MR (2006). Child maltreatment and the developing HPA axis. Horm Behav 50: 632-639.

Timbremont B, Braet C (2002). Manual for the Dutch Children's Depression Inventory. Swets \& Zeitlinger: Lisse, the Netherlands.

Thompson C, Syddall H, Rodin I, Osmond C, Barker DJP (2001). Birth weight and the risk of depressive disorder in late life. Brit J Psychiat 179: 450-455.

Van den Bergh BRH (1992). Maternal emotions during pregnancy and fetal and neonatal behaviour. In: Nijhuis JG (ed). Fetal Behaviour. Developmental and Perinatal Aspects. Oxford University Press: Oxford, UK. pp 157-174.

Van den Bergh BRH, Marcoen A (2004). High antenatal maternal anxiety is related to ADHD symptoms, externalizing problems and anxiety in 8/9-year-olds. Child Dev 75: 1085-1097.

Van den Bergh BRH, Mulder EJH, Mennes M, Glover V (2005a). Antenatal maternal anxiety and stress and the neurobehavioral development of fetus and child: links and possible mechanisms. A review Neurosci Biobehav Rev 29: 237-258.

Van den Bergh BRH, Mennes M, Oosterlaan J, Stevens V, Stiers P, Marcoen A et al (2005b). High antenatal maternal anxiety is related to impulsivity during performance on cognitive tasks in 14- and 15-year-olds. Neurosci Biobehav Rev 29: 259-269.

Van den Bergh BRH, Mennes M, Stevens V, van der Meere J, Börger N, Stiers P et al (2006). ADHD deficit as measured in adolescent boys with a continuous performance task is related to antenatal maternal anxiety. Pediatric Research 59: 78-82.

Van Den Eede F, Van Broeckhoven C, Claes SJ (2005). Corticotropin-releasing factor-binding protein, stress and major depression. Ageing Res Rev 4: 213-239.

Van der Ploeg HM, Defares PB, Spielberger CD (1980). Manual of the Self-Evaluation Questionnaire: a Dutch Version of the State-Trait Anxiety Inventory. Swets \& Zeitlinger: Lisse, the Netherlands.

van Praag HM, de Kloet ER, van Os J (2004). Stress, the Brain and Depression. Cambridge University Press: Cambridge.

van West D, Van Den Eede F, Del-Favero J, Souery D, Norrback K-F, Van Duijn C et al (2006). Glucocorticoid receptor genebased SNP analysis in patients with recurrent major depression. Neuropsychopharmacology 31: 620-627.

Weaver ICG, La Plante P, Weaver S, Parent A, Sharma S, Diorio J et al (2001). Early environmental regulation of hippocampal glucocorticoid receptor gene expression: Characterization of intracellular mediators and potential genomic target sites. Mol Cell Endocrinol 185: 205-218.

Weaver ICG, Champagne FA, Brown SE, Dymov S, Sharma S, Meaney MJ et al (2005). Reversal of maternal programming of stress responses in adult offspring through methyl supplementation: altering epigenetic marking later in life. J Neurosci 25: 11045-11054.

Welberg LAM, Seckl JR (2001). Prenatal stress, glucocorticoids and the programming of the brain. J Neuroendocrinol 13: 113-128.

Wüst S, Federenko I, Hellhamer DH, Kirschbaum C (2000). Genetic factors, perceived chronic stress, and the free cortisol response to awakening. Psychoneuroendocrinology 25: 707-720.

Young EA, Vazquez D, Jiang H, Pfeffer CR (2006). Saliva cortisol and response to dexamethasone in children of depressed parents. Biol Psychiatry 60: 831-836. 